\title{
THE LARGE RANGE OF DARK MATTER CONTENT IN DWARF GALAXIES AND ITS IMPLICATIONS
}

\section{S.A. PUSTILNIK AND V.A. LIPOVETSKY}

Special Astrophysical Observatory, Russian AS

J.-M. MARTIN

Observatoire de Paris-Meudon, ARPEGES

AND

T.X. THUAN

Astronomy Department, University of Virginia

We present the analysis of a new set of radio and optical observations of a large sample of Byurakan Blue Compact Galaxies. HI spectra were obtained with the Nançay 300-m and Green Bank 43-m radio telescopes. CCD-images were taken with the KPNO 0.9-m and Whipple Observatory $1.2-\mathrm{m}$ telescopes. Dark Matter (DM) to luminous mass ratios in these BCGs were found to vary from about less than 0.5 up to 14 . Recent data taken from the literature indicate this same range. This result has important consequences on models of dwarf galaxy formation, indicating possibly different formation mechanisms. The standard CDM model of dwarfs formation requires large DM halos. However the formation of dwarfs as tidal debris resulting from strong interactions of massive spirals leads naturally to dwarfs with low content of DM. On Fig. 1 we show DM to luminous mass ratio versus rotational velocity for our BCG and some other galaxies.

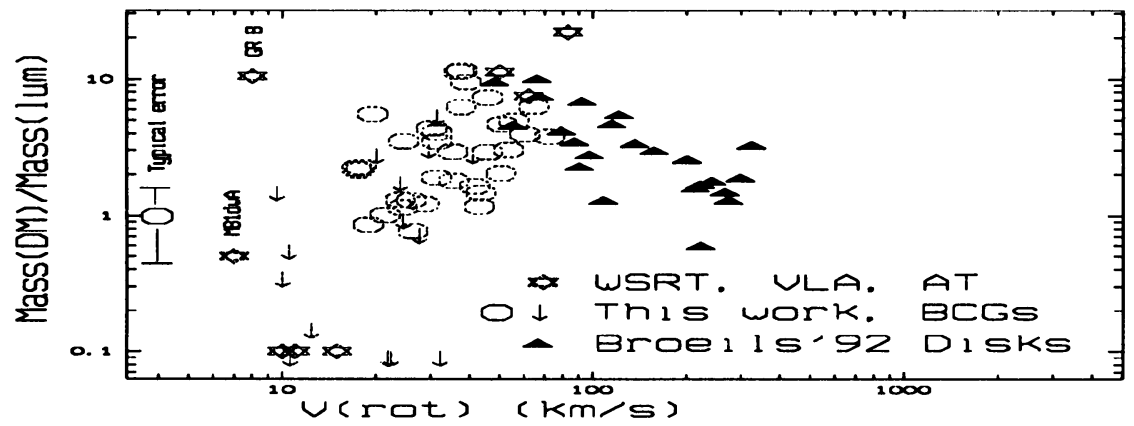

